\title{
Evaluation of Autogenous Engineered Septal Cartilage Grafts in Rabbits: A Minimally Invasive Preclinical Model
}

\author{
Anton Kushnaryov, ${ }^{1,2}$ Tomonoro Yamaguchi, ${ }^{3}$ Kristen K. Briggs, \\ Van W. Wong, ${ }^{4}$ Marsha Reuther, ${ }^{1,2}$ Monica Neuman, ${ }^{5}$ Victor Lin, ${ }^{6}$ \\ Robert L. Sah, ${ }^{4}$ Koichi Masuda, ${ }^{3}$ and Deborah Watson ${ }^{1,2}$ \\ ${ }^{1}$ Division of Otolaryngology-Head and Neck Surgery, University of California, 3350 La Jolla Village Drive, 112-C, San Diego, \\ CA 92161, USA \\ ${ }^{2}$ Head and Neck Surgery Section, VA San Diego Healthcare System, San Diego, CA 92161, USA \\ ${ }^{3}$ Department of Orthopedic Surgery, University of California, San Diego, La Jolla, CA 92161, USA \\ ${ }^{4}$ Department of Bioengineering, University of California, San Diego, La Jolla, CA 92161, USA \\ ${ }^{5}$ Creighton University School of Medicine, Omaha, NE 68102, USA \\ ${ }^{6}$ University of North Texas Health Science Center, Fort Worth, TX 76107, USA \\ Correspondence should be addressed to Anton Kushnaryov; akushnaryov@ucsd.edu
}

Received 18 April 2014; Revised 11 June 2014; Accepted 12 June 2014; Published 23 July 2014

Academic Editor: Samy Elwany

Copyright ( $) 2014$ Anton Kushnaryov et al. This is an open access article distributed under the Creative Commons Attribution License, which permits unrestricted use, distribution, and reproduction in any medium, provided the original work is properly cited.

\begin{abstract}
Objectives. (1) Evaluate safety of autogenous engineered septal neocartilage grafts and (2) compare properties of implanted grafts versus controls. Study Design. Prospective, basic science. Setting. Research laboratory. Methods. Constructs were fabricated from septal cartilage and then cultured in vitro or implanted on the nasal dorsum as autogenous grafts for 30 or 60 days. Rabbits were monitored for local and systemic complications. Histological, biochemical, and biomechanical properties of constructs were evaluated. Results. No serious complications were observed. Implanted constructs contained more DNA $(P<0.01)$ and less sGAG perDNA $(P<0.05)$ when compared with in vitro controls. Confined compressive aggregate moduli were also higher in implanted constructs $(P<0.05)$ and increased with longer in vivo incubation time $(P<0.01)$. Implanted constructs displayed resorption rates of 20-45 percent. Calcium deposition in implanted constructs was observe. Conclusion. Autogenous engineered septal cartilage grafts were well tolerated. As seen in experiments with athymic mice, implanted constructs accumulated more DNA and less sGAG when compared with in vitro controls. Confined compressive aggregate moduli were higher in implanted constructs. Implanted constructs displayed resorption rates similar to previously published studies using autogenous implants of native cartilage.
\end{abstract}

\section{Introduction}

Craniofacial defects created from tumor resection, traumatic loss, or congenital deformities frequently require complex surgical reconstruction to rebuild missing cartilage and bony support. A variety of autologous, allogeneic, and synthetic grafts have traditionally been used to provide structural support in these procedures [1-4]. While certainly useful in specific situations, each grafting material has drawbacks. Allogeneic grafts carry the risks of immune rejection and the potential for disease transmission. The use of synthetic materials can be complicated by infection and extrusion.
Anecdotally, autologous grafts are favored by many reconstructive surgeons. Cartilaginous autografts are a commonly used structural support medium; various donor sites including rib, auricle, and nasal septum are all currently in use [5-7]. Nasal septal cartilage offers important advantages over other cartilage types due to its favorable mechanical properties, ease of harvest, and minimal donor site morbidity. However, its use remains limited by several factors. These include the limited amount of tissue available and a predetermined, potentially suboptimal, semirigid geometric structure. Tissue engineering of autologous neocartilage offers the potential to generate abundant amounts of cartilage from a small donor 
specimen, with the ability to create grafts in a variety of customizable shapes and sizes.

Previous studies have demonstrated the ability to successfully create human septal neocartilage constructs from single and pooled donor specimens [8-11]. Homicz et al. successfully generated human nasal septal neocartilage constructs from chondrocytes seeded onto a polyglycolic acid scaffold [8]. Further studies have refined septal cartilage tissue bioengineering techniques to allow growth of semirigid threedimensional constructs from single donor samples using a novel Alginate Recovery Method (ARC) [9-11]. Recent research has evaluated the feasibility of implanting these constructs in a murine animal model. Chang et al. implanted human nasal septal neocartilage constructs into athymic mice and evaluated biocompatibility and maturation after 30 or 60 days in vivo. The grafts demonstrated survival and in vivo maturation as evidenced by increased mechanical strength, DNA content, and distinct morphological characteristics not seen in nonimplanted controls [12].

At the same time as techniques in the field of tissue engineering, including tissue engineering of nasal septal constructs, were elaborated and refined, a parallel area of research involved animal models of human facial defects developed. The New Zealand White Rabbit has been used as an animal model for decades as a human surrogate to examine a variety of pathologies encountered by the reconstructive surgeon. This animal model has been used to examine facial growth and development [13-16], as well as in evaluating facial implants [17, 18]. Additionally, de Souza et al. used the New Zealand White Rabbit in experiments comparing crushed and uncrushed septal cartilage implants and the effect of crushing on calcification, resorption, and viability [19]. They found that cartilage, whether autologous or homologous, crushed or uncrushed, demonstrated viability, although fibrous ingrowth was noted when crushed cartilage was implanted. Ścierski et al. also used this rabbit model to investigate the efficacy of various synthetics used for repair of nasal septal perforations [20]. In 2001, Wong et al. described an invasive method of rabbit nasal septal cartilage harvest and characterized specific cellular, metabolic, and physical properties of the cartilage [21].

Although investigators have used the New Zealand White Rabbit as an animal model and extrapolated these results to humans, no studies to date have used autologous tissue engineered neocartilage constructs in their study design, nor has any study evaluated the feasibility and safety of these constructs. The current study was designed to establish a preclinical animal model for the use of autologous nasal septal neocartilage grafts. Demonstration of safety and efficacy in an animal model is an important prerequisite for undertaking any studies with this technology in humans.

\section{Methods}

2.1. Creation of Rabbit Nasal Septal Defect and Harvest of Cartilaginous Septum. A $5 \times 10 \mathrm{~mm}$ portion of nasal septum was harvested from sixteen adult female New Zealand White Rabbits near the nasal dorsum according to the following surgical technique: after sedation and intubation the rabbit was placed prone on a heated surgical table. An area of $2 \times 4 \mathrm{~cm}$ on the midline nasal dorsum was shaved with electric clippers, prepped with povidone iodide solution, and sterilely draped. A $15 \mathrm{~mm}$ midline supraperiosteal incision was made over the dorsum. A $20 \times 15 \mathrm{~mm}$ periosteal flap was raised sharply. An osteotome was used to raise a $10 \times$ $20 \mathrm{~mm}$ bone flap which was left anchored rostrally. The upper lateral cartilages were separated from the dorsal nasal septum in continuity with a mucoperichondrial flap. The exposed cartilage was harvested sharply. The upper lateral cartilages were replaced. The bone and periosteal flaps were replaced and the incision closed in layers. During the procedure, each animal's heart rate, respiratory rate, oxygen saturation, reflexes, and response to pain were monitored. The length of the procedure was 15-25 minutes per rabbit. All animals were subject to ongoing general health monitoring according to UCSD Institutional Animal Care and Use Committee guidelines.

2.2. Creation of Cartilage Constructs. Each septal specimen was used to fabricate 2-3 autologous neocartilage constructs according to a previously published protocol using the ARC method [10]. Although this has been described briefly, the method is briefly summarized here: the samples we obtain during surgery are cleaned and diced. The chondrocyte fraction is isolated and ultimately plated in a tissue culture flask as a monolayer, using a proprietary culture medium. When the cells are confluent, they are released from the tissue culture flask and suspended in a solution containing low viscosity Alginate. Slowly dripping this solution into a second solution creates 3-dimensional chondrocyte containing Alginate beads. These beads are further cultured and eventually depolymerized. In the final step, free chondrocytes, and the extracellular matrix they have been secreting, are further cultured and matured in tissue wells and become neocartilage constructs. After a culture period of 90 days, the constructs were harvested. Each construct was about $15 \mathrm{~mm}$ in diameter by about $2-4 \mathrm{~mm}$ thick at the time of harvest. Half of the samples remained in culture as in vitro controls. The remaining constructs were implanted as autologous dorsal onlay grafts for a period of 30 or 60 days.

\subsection{Implantation as Autologous Nasal Dorsal Onlay Grafts.} After confirming adequate sedation, each rabbit was placed prone on a heated surgical table. The nasal dorsum was shaved with electric clippers, prepped with povidone iodide solution, and sterilely draped. A $15 \mathrm{~mm}$ midline incision over the dorsum was made to a supraperiosteal depth. Blunt dissection generated a precise pocket for implantation. One or two constructs were placed into separate pockets over the rabbit nasal dorsum. The skin and subcutaneous tissue were approximated using resorbable suture. During the procedure, the animal was monitored using the same protocol as the initial surgical procedure. The length of the procedure was 10-15 minutes per animal. On the appropriate experimental day, the animals were sacrificed and each neocartilage construct was recovered for analysis. 
2.4. Biochemical Evaluation. A small portion of the neocartilage constructs was digested with proteinase $\mathrm{K}$ (PK) (Roche Diagnostics, Indianapolis, IN) in phosphate-buffered EDTA for 16 hours. The sGAG content in the PK digests was determined using the dimethyl-methylene blue (DMMB) reaction according to prior published reports [22]. GAG content was normalized per gram of wet tissue weight (before digestion) and to DNA content [23]. The hydroxyproline assay was used to determine the amount of total collagen in the PK digests according to a previously published protocol [23]. Hydroxyproline content was normalized per gram of wet tissue weight (prior to digestion) and to DNA content. The amount of collagen in the sample was estimated using the formula: Collagen content was estimated using the following relationship: $1 \mathrm{~g}$ of hydroxyproline is present in $7.69 \mathrm{~g}$ of collagen $(\mathrm{g})$. The PicoGreen DNA assay was used to determine the amount of DNA in the PK digests according to previously published protocols [24].

2.5. Histochemistry. A portion of each construct was placed in OCT compound, frozen by immersion in liquid nitrogencooled isopentane, and sectioned using a Cryostat (Leica Biosystems, Wetzlar, Germany). The 30-40 micron samples were mounted on poly-L lysine coated slides (Polysciences Inc., Warrington, PA).

Immunohistochemistry was performed with the Vectastain Elite ABC kit (Vector Laboratories, Burlingame, CA), a peroxidase-based detection system, according to the manufacturer's instructions. The samples were probed with one of three antibodies: anti-type I collagen (number ab6308, Abcam, Cambridge, MA) at a concentration of $7 \mu \mathrm{g} / \mathrm{mL}$, antitype II collagen (number 7005, Chondrex Inc., Redmond, WA) at a concentration of $0.5 \mu \mathrm{g} / \mathrm{mL}$, or a mouse nonspecific IgG used at $7 \mu \mathrm{g} / \mathrm{mL}$ as a negative control. Sections were counterstained with methyl green nuclear stain (Vector Laboratories, Burlingame, CA).

To visualize GAG deposition, additional sectioned samples were stained with $0.1 \%$ Alcian Blue using standard techniques. Alizarin Red staining was performed to visualize calcium deposition in the sectioned samples according to standard techniques. Samples were documented by photomicroscopy.

2.6. Confined Compression Testing. Specimens for mechanical testing were kept hydrated with phosphate buffered saline (PBS) supplemented with calcium, magnesium, and protease inhibitors during testing. The thickness of each construct was measured at 3 points distributed around the circumference and center of each sample, using a noncontact laser displacement sensor $( \pm 0.024 \mathrm{~mm}$ resolution).

For compression testing, a $4.8 \mathrm{~mm}$ diameter disk was isolated from each construct and tested following a previously published protocol [25]. The compressive mechanical properties of cartilage samples were calculated from the acquired data assuming tissue homogeneity. The equilibrium-confined compression (aggregate) modulus HA0 was estimated by using a least-squares method to fit the equilibrium stressstrain data to a finite deformation constitutive relation, which has been shown previously to provide an excellent fit of compression data [24-27]. The permeability $\mathrm{kp}$ at $15 \%$ compressive strain was estimated by fitting the measured dynamic stiffness (amplitude and phase) to the theoretical solution using the computed value of $\mathrm{HA}$ at $15 \%$ compressive strain.

2.7. Statistical Analysis. Statistical analysis was performed using Systat 10.2 (SYSTAT Software Inc., Richmond, CA). Means are presented \pm the standard deviation (SD). Differences in DNA, GAG, collagen, and confined compression modulus were assessed using a one-way analysis of variance (ANOVA) to determine the fixed effect of maturation time. If ANOVA identified an overall significant effect, post hoc Tukey's honestly significant differences (HSD) tests were used to identify significant differences between time points. A $P \leq$ 0.05 was considered significant.

\section{Results}

Nasal septal cartilage specimens from sixteen New Zealand White Adult Rabbits were harvested using a minimally invasive surgical technique. The cartilage was used to generate tissue engineered neocartilage constructs which were matured in vitro using the ARC method. One half of these constructs were implanted as autologous nasal dorsal onlay grafts and examined after 30 or 60 days, while the other half served as in vitro controls. Histological, biochemical, and biomechanical data were collected for the two onlay graft cohorts and compared with the data collected for the neocartilage constructs which remained as in vitro controls.

There was a significant increase in the total DNA content by weight in the in vivo neocartilage constructs when compared with the in vitro control constructs, at both 30 and 60 days postimplantation. In the 30 day group, the mean DNA content per wet weight was $0.95 \pm 0.45 \mu \mathrm{g} / \mathrm{mg}$ and $0.27 \pm$ $0.10 \mu \mathrm{g} / \mathrm{mg}$ for the in vivo and in vitro groups, respectively (Figure $1(\mathrm{a}), P=0.00)$. Similarly, the mean DNA content per wet weight for the in vivo and in vitro groups at 60 days postimplantation was $0.76 \pm 0.33 \mu \mathrm{g} / \mathrm{mg}$ and $0.23 \pm$ $0.12 \mu \mathrm{g} / \mathrm{mg}$, respectively (Figure $1(\mathrm{a}), P=0.00$ ).

In contrast, sGAG deposition per DNA decreased in the in vivo constructs when compared with in vitro control constructs, at both 30 and 60 days. At 30 days postimplantation, the mean sGAG per DNA was $5.70 \pm 3.46 \mu \mathrm{g} / \mu \mathrm{g}$ and $29.5 \pm$ $15.7 \mu \mathrm{g} / \mu \mathrm{g}$ for the in vivo and in vitro groups, respectively (Figure 1(b), $P=0.02$ ). At 60 days post implantation, the in vivo groups had $4.27 \pm 1.10 \mu \mathrm{g} / \mu \mathrm{g}$ of sGAG/DNA and the in vitro groups had $38.8 \pm 26.5 \mu \mathrm{g} / \mu \mathrm{g}$ of sGAG/DNA, showing the same trend as the 30 day cohort (Figure $1(b), P=0.00$ ). In contrast, the total collagen content per DNA remained stable between the in vivo and in vitro cohorts, at 30 and 60 days postimplantation (data not shown).

Confined compression tests were performed to determine the aggregate modulus (HA0) and the hydraulic permeability $(\mathrm{k} 0)$ of each of the neocartilage constructs. HA0 is a measure of the tissue stiffness at equilibrium. The higher the HA0 is, the less the tissue deforms under a given load. HA0 


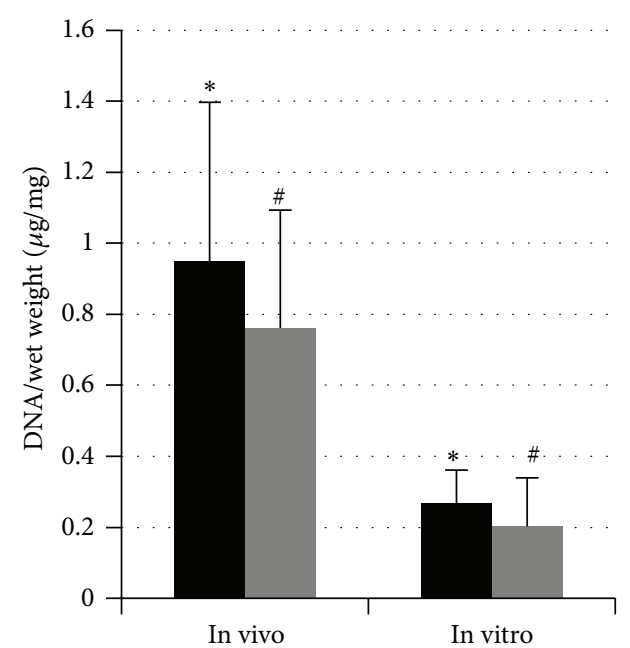

(a)

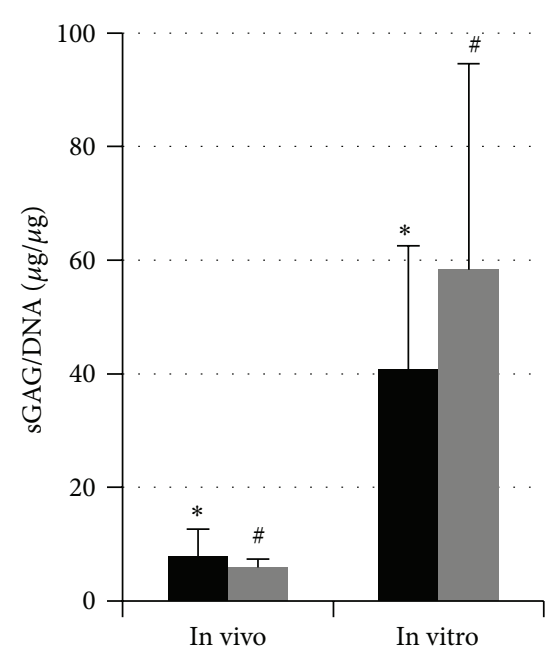

(b)

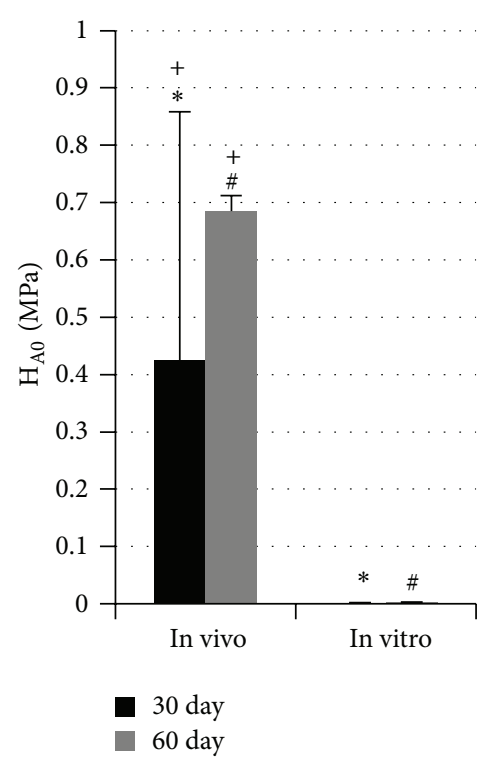

(c)

FIGURE 1: Biochemical and biomechanical characterization of implanted and control constructs after 30 or 60 days. Implanted in vivo constructs accumulated more DNA per wet weight (a) and less sGAG per DNA (b) than the in vitro control constructs. The aggregate modulus (HA0) was also greater for implanted constructs when compared with the in vitro control constructs (c). In addition, HA0 increased upon increasing time of in vivo incubation (d). ${ }^{*} P<0.05,{ }^{\#} P<0.01$, and ${ }^{+} P=0.008$.

values increased in the in vivo neocartilage constructs when compared with the in vitro control constructs at both 30 and 60 days postimplantation. At 30 days postimplantation, HA0 values were $0.263 \pm 0.203 \mathrm{MPa}$ and $0.001 \pm 0.001 \mathrm{MPa}$ for the in vivo and in vitro groups, respectively (Figure 1(c), $P=0.021$ ). After 60 days postimplantation, HA0 values increased to $0.686 \pm 0.027 \mathrm{MPa}$ and $0.002 \pm 0.001 \mathrm{MPa}$ for the in vivo and in vitro groups, respectively (Figure 1(c), $P=0.001)$. In addition, there was a significant increase in HA0 with increasing in vivo incubation time (Figure $1(\mathrm{c}$ ), $P=0.008)$. The transient mechanical behavior of cartilage is related to its interstitial fluid flow which is governed by hydraulic permeability $(\mathrm{k} 0)$. In this study, $\mathrm{k} 0$ values at 15 , 30 , and $45 \%$ compression were not significantly different between the neocartilage construct groups (data not shown).

Histologic examination of the neocartilage constructs showed a distribution of chondrocytes in lacunae within an extracellular matrix, typical of native cartilage [28]. Histochemical analyses of neocartilage tissue showed deposition of sGAG in both in vitro and in vivo tissue sections (data not shown). In addition, all constructs accumulated type I and type II collagen (Figure 2). Neocartilage constructs incubated in vivo for 30 or 60 days also formed enlarged lacunae containing large numbers of cells (Figures 2 and 3 ). Furthermore, in vivo incubation of the neocartilage constructs resulted in diffuse calcium deposition as visualized by Alizarin Red S staining (Figure 3 ) and microtomography analyses (data not shown). Light microscopy revealed no evidence of inflammatory infiltrate in the specimens, or gross evidence of a rabbit inflammatory response at any time postoperatively. No ingrowth from the surrounding tissues was observed. The degree of calcification varied from $20 \%$ to
$45 \%$. In vivo and In vitro specimens at 30 and 60 days are shown in Figure 4.

There were no serious complications in any of the rabbits studied. Out of sixteen rabbits, two had transient, self-limited elevation of the nasal skin flap with expiration after the initial surgical procedure. This resolved after 1-2 days and produced no long-term sequelae. There were no postoperative respiratory problems, no surgical site infections or dehiscence, and no other adverse events associated with this experiment.

\section{Discussion}

In this study we demonstrated successful in vivo implantation and maturation of autologous nasal septal neocartilage constructs. To our knowledge, this is the first reported animal model system for the implantation of wholly autologous tissue engineered cartilage. Previous studies have evaluated autologous native cartilage grafts in animal model systems $[19,29]$. Bermueller et al. recently published an article describing tissue engineered cartilage used in orthotopic rabbit nasal septal defects [30]. These constructs used autologous chondrocytes and nonautologous marine collagen scaffolding. Other studies have used implanted human septal chondrocyte-derived constructs in an immune deficient murine model [12]. Another recent study by Fulco et al. described the implantation of autologous tissue engineered cartilage in the humans; however, these constructs were created by embedding chondrocytes in an off-the-shelf, nonautologous collagen matrix [31]. By using autologous tissue engineered construct implants, our study serves as a foundation for future studies in animals, leading towards 


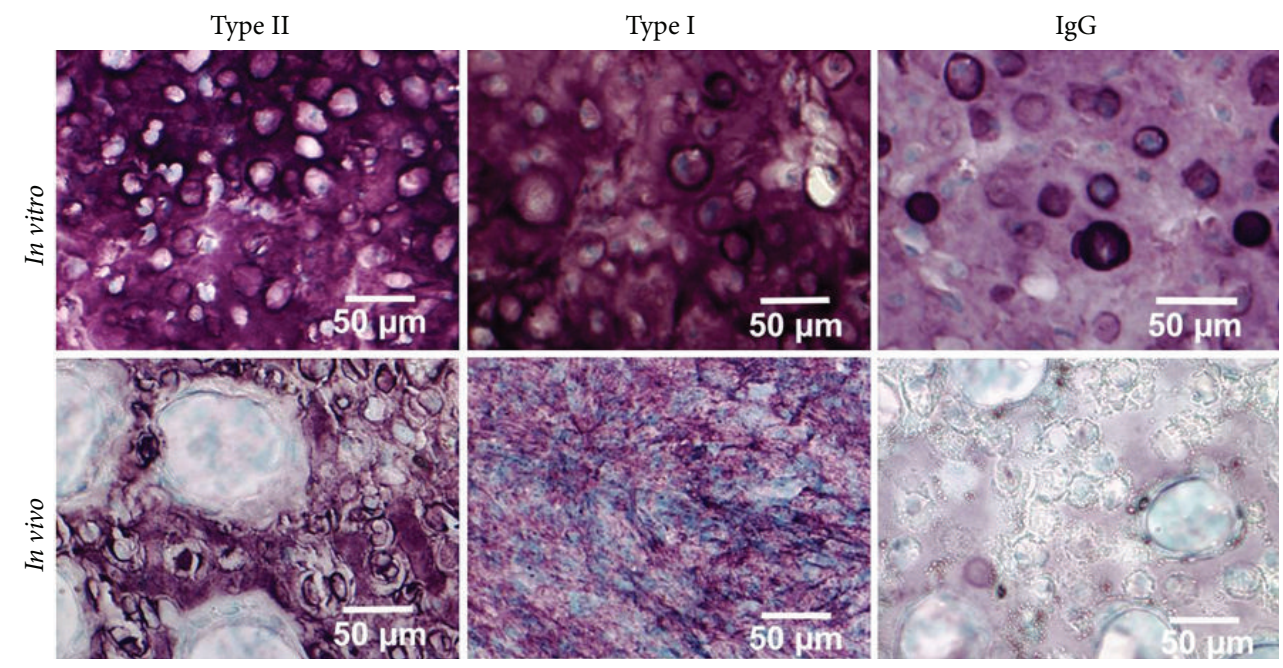

FIGURE 2: Immunohistochemical staining for type I and type II collagen in in vitro control neocartilage constructs (top) or after 30 days in vivo (below). Sections were counterstained with the nuclear stain methyl green. Neocartilage constructs had robust staining for both collagen types II and I (left and middle) compared with the nonspecific control IgG (right). In addition, in vivo tissue had enlarged lacunae containing large clusters of cells.

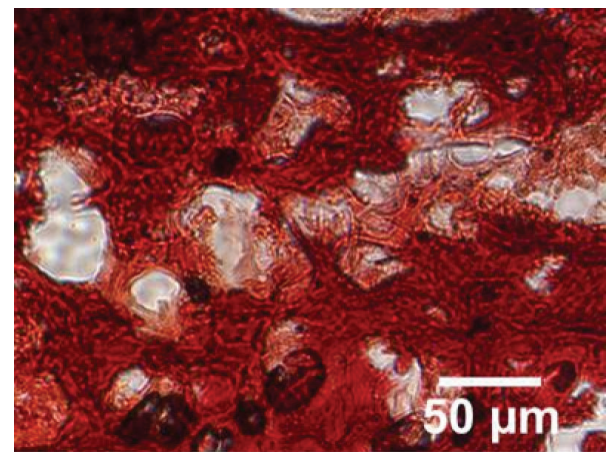

FIgURE 3: Alizarin Red S staining confirmed deposition of calcium in neocartilage constructs after 30 days implantation.

eventual in vivo human studies of wholly autologous tissue engineered neocartilage grafts.

To date, the literature also does not report any detailed, reproducible, minimally invasive surgical approach through the nasal vault for harvesting septal cartilage. Gubisch et al. have described a more invasive technique involving multiple incisions including hemitransfixion incisions [28]. Wong et al. described an approach which involved a larger skin incision, more extensive dissection, and the use of power instrumentation to create a larger bone flap [21]. Using the minimally invasive technique described in the current study, the rabbits displayed no significant respiratory issues or long-term postoperative deficits. In addition, no surgical site infections or wound dehiscence occurred. This study serves to establish the feasibility of a minimally invasive surgical procedure, using the New Zealand White rabbit as an animal model, for autologous tissue engineered nasal septal neocartilage grafts. It also demonstrates the safety of the reimplanted neocartilage constructs. No adverse events were observed in either experimental group. The postsurgical implant sites did not show evidence of graft rejection, infection, capsule formation, or other undesired features.

DNA content, a surrogate measure of cell number, was higher in the in vivo than in the in vitro neocartilage constructs at both 30 and 60 days postimplantation. We postulate that the increase in DNA in the in vivo group reflects a change in the constructs reflecting increased cellularity associated with maturation in the complex environment within the rabbit. In contrast sGAG accumulation per DNA, a proxy for the amount of proteoglycan contained in cartilage tissue, decreased significantly after in vivo implantation. This correlates with the increased DNA content seen in the in vivo constructs. An increase in DNA and decrease in sGAG content were also observed when tissue engineered human nasal septal neocartilage constructs were subjected to a period of maturation in vivo after implantation in nude mice [12]. In contrast to the nude mouse implantation study, in vivo maturation did not enhance collagen levels in the current work. Differences in collagen levels between these two studies may be due to the methods used to measure collagen levels. In the current study, the hydroxyproline assay was used to measure total collagen, while ELISA was used to determine solubilized type II collagen in the nude mouse study. Another explanation for the differences in collagen level between the two studies may be the presence of calcium deposition and chondrocyte hypertrophy observed in the implanted rabbit neocartilage tissue.

Implantation of autologous neocartilage constructs resulted in significant gains in mechanical stiffness. The aggregate moduli (HA0) increased in the in vivo neocartilage constructs when compared with the in vitro control constructs at both 30 and 60 days postimplantation. Additional gains in stiffness were observed with increasing time of in vivo incubation.

Histological examination of the autologous tissue engineered neocartilage constructs revealed chondrocytes nestled 

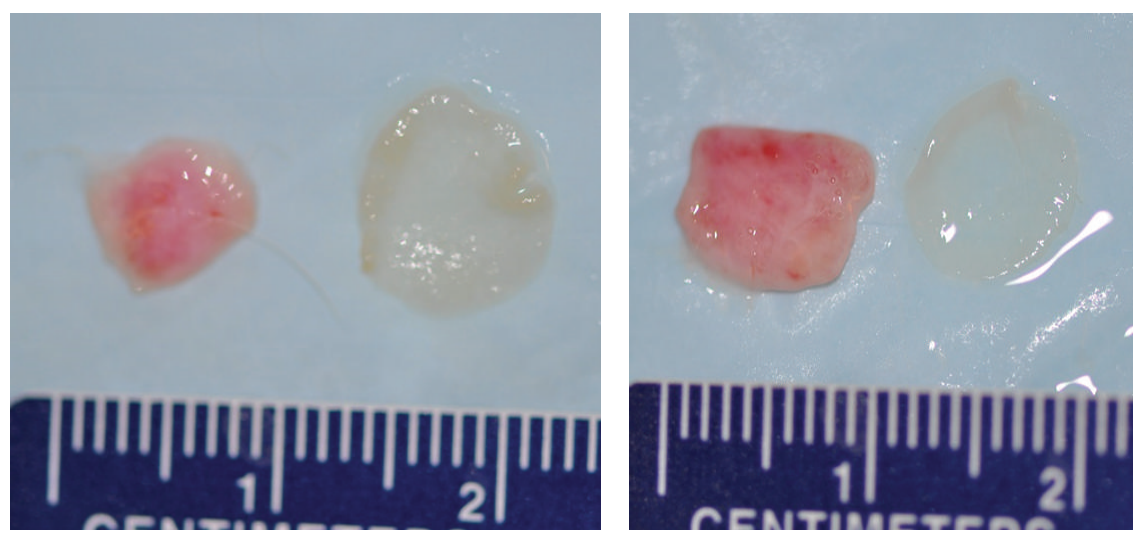

FIGURE 4: Representative photographs of in vivo and in vitro constructs at 30 and 60 days are shown. Measurements are in centimeters.

in lacunae and distributed within an extracellular matrix as seen in native nasal septal cartilage [32]. Alcian blue staining of in vitro and in vivo incubated neocartilage constructs showed accumulation of GAG at 30 and 60 days postimplantation and all neocartilage constructs expressed both type I and type II collagen. It was noted, however, that diffuse calcium deposition and minor resorption based on construct diameter in the in vivo implants were present. Previously published research by Lattyak et al. demonstrated resorption rates of up to $30 \%$ in rabbits implanted with autologous septal cartilage [29]. They implanted autologous septal, auricular and costal cartilage as dorsal onlay grafts and observed resorption rates of $30.8 \%$ in septal cartilage, $21.3 \%$ in auricular cartilage, and $7.6 \%$ in costal cartilage. Another study by de Souza et al. evaluating rabbit autologous septal grafts demonstrated no calcification of the graft but did report resorption rates of $15-50 \%$ [19]. Since this study is the first report demonstrating implantation of autologous rabbit septal cartilage constructs, it is difficult to compare rates of calcification and resorption to experiments using crushed or uncrushed native rabbit septal cartilage. Furthermore, it is unknown whether this resorption would be balanced by the formation of a fibrous capsule as has been suggested by some authors $[19,33]$.

This study has demonstrated a reliable noninvasive surgical approach to the rabbit septum and the utility of the rabbit model. This can be very useful in future investigations to evaluate the longevity of tissue engineered constructs in the in vivo setting.

\section{Conflict of Interests}

The authors declare that there is no conflict of interests regarding the publication of this paper.

\section{Acknowledgment}

This material is based upon work supported in part by the Department of Veterans Affairs, Veterans Health Administration, Office of Research and Development (BL R\&D) MeritAward (Deborah Watson), NIH R01
AR044058 (Robert L. Sah) and the American Academy of Otolaryngology-Head and Neck Surgery Foundation Resident Research Award (Anton Kushnaryov). This project was presented at the annual meeting of the American Academy of Otolaryngology-Head and Neck Surgery, Vancouver, BC, 2013.

\section{References}

[1] T. Berling and P. Runeson, "Efficient evaluation of multifactor dependent system performance using fractional factorial design," IEEE Transactions on Software Engineering, vol. 29, no. 9, pp. 769-781, 2003.

[2] M. E. Tardy Jr., J. Denneny III, and M. H. Fritsch, "The versatile cartilage autograft in reconstruction of the nose and face," Laryngoscope, vol. 95, no. 5, pp. 523-533, 1985.

[3] J. Komender, W. Marczynski, D. Tylman, H. Malczewska, A. Komender, and D. Sladowski, "Preserved tissue allografts in reconstructive surgery," Cell and Tissue Banking, vol. 2, no. 2, pp. 103-112, 2001.

[4] D. B. Lovice, M. D. Mingrone, and D. M. Toriumi, "Grafts and implants in rhinoplasty and nasal reconstruction," Otolaryngologic Clinics of North America, vol. 32, no. 1, pp. 113-141, 1999.

[5] A. Moretti and S. Sciuto, "Rib grafts in septorhinoplasty," Acta Otorhinolaryngologica Italica, vol. 33, no. 3, pp. 190-195, 2013.

[6] D. Son, M. Kwak, S. Yun, H. Yeo, J. Kim, and K. Han, "Large auricular chondrocutaneous composite graft for nasal alar and columellar reconstruction," Archives of Plastic Surgery, vol. 39, no. 4, pp. 323-328, 2012.

[7] J. Lin, X. Chen, X. Wang et al., "A modified septal extension graft for the asian nasal tip," JAMA Facial Plastic Surgery, vol. 15, no. 5, pp. 362-368, 2013.

[8] M. R. Homicz, B. L. Schumacher, R. L. Sah, and D. Watson, "Effects of serial expansion of septal chondrocytes on tissueengineered neocartilage composition," Otolaryngology-Head and Neck Surgery, vol. 127, no. 5, pp. 398-408, 2002.

[9] K. Masuda, T. Miyazaki, B. Pfister, E. Horvath, and E. J.-M. Thonar, "Human tissue engineered cartilage by the alginaterecovered-chondrocyte method after an expansion in monolayer," Transactions of the Orthopaedic Research Society, vol. 27, article 467, 2002. 
[10] K. Masuda, R. L. Sah, M. J. Hejna, and E. J. Thonar, "A novel two-step method for the formation of tissue-engineered cartilage by mature bovine chondrocytes: the alginate-recoveredchondrocyte (ARC) method," Journal of Orthopaedic Research, vol. 21, no. 1, pp. 139-148, 2003.

[11] S. H. Chia, B. L. Schumacher, T. J. Klein et al., "Tissueengineered human nasal septal cartilage using the alginaterecovered-chondrocyte method," Laryngoscope, vol. 114, no. 1, pp. 38-45, 2004.

[12] A. A. Chang, M. S. Reuther, K. K. Briggs et al., "In vivo implantation of tissue-engineered human nasal septal neocartilage constructs: A pilot study," Otolaryngology-Head and Neck Surgery, vol. 146, no. 1, pp. 46-52, 2012.

[13] G. J. Nolst Trenite, C. D. A. Verwoerd, and H. L. VerwoerdVerhoef, "Reimplantation of autologous septal cartilage in the growing nasal septum. I. The influence of resection and reimplantation of septal cartilage upon nasal growth: an experimental study in rabbits," Rhinology, vol. 25, no. 4, pp. 225-236, 1987.

[14] G. J. Nolst Trenite, C. D. A. Verwoerd, and H. L. VerwoerdVerhoef, "Reimplantation of autologous septal cartilage in the growing nasal septum. II. The influence of reimplantation of rotated or crushed autologous septal cartilage on nasal growth: an experimental study in growing rabbits," Rhinology, vol. 26, no. 1, pp. 25-32, 1988.

[15] C. D. A. Verwoerd, N. A. M. Urbanus, and D. C. Nijdam, "The effects of septal surgery on the growth of nose and maxilla," Rhinology, vol. 17, no. 2, pp. 53-63, 1979.

[16] C. D. A. Verwoerd, N. A. M. Urbanus, and G. J. Mastenbroek, "The influence of partial resections of the nasal septal cartilage on the growth of the upper jaw and the nose: an experimental study in rabbits," Clinical Otolaryngology and Allied Sciences, vol. 5, no. 5, pp. 291-302, 1980.

[17] B. Kramp, H. E. Bernd, W. A. Schumacher et al., "Poly- $\beta$ hydroxybutyric acid (PHB) films and plates in defect covering of the osseus skull in a rabbit model," Laryngo- Rhino- Otologie, vol. 81, no. 5, pp. 351-356, 2002.

[18] R. V. Oliveira, L. S. de Souza Nunes, H. N. Filho, L. de Andrade Holgado, D. A. Ribeiro, and M. A. Matsumoto, "Fibrovascularization and osteogenesis in high-density porous polyethylene implants," Journal of Craniofacial Surgery, vol. 20, no. 4, pp. 1120-1124, 2009.

[19] M. M. A. de Souza, L. C. Gregório, R. Sesso, A. S. Souza, and F. Settanni, "Study of rabbit septal cartilage grafts placed on the nasal dorsum," Archives of Facial Plastic Surgery, vol. 10, no. 4, pp. 250-254, 2008.

[20] W. Ścierski, A. Polok, G. Namyslowski et al., "Ocena wybranych biomateriałów do rekonstrukcji perforacji przegrody nosa," Otolaryngologia Polska, vol. 61, no. 5, pp. 842-846, 2007.

[21] B. J. F. Wong, K. K. H. Chao, H. K. Kim et al., "The porcine and lagomorph septal cartilages: models for tissue engineering and morphologic cartilage research," The American Journal of Rhinology, vol. 15, no. 2, pp. 109-116, 2001.

[22] R. W. Farndale, D. J. Buttle, and A. J. Barrett, "Improved quantitation and discrimination of sulphated glycosaminoglycans by use of dimethylmethylene blue," Biochim Biophys Acta, vol. 883, no. 2, pp. 173-177, 1986.

[23] D. S. Jackson and E. G. Cleary, "The determination of collagen and elastin," Methods of Biochemical Analysis, vol. 15, pp. 25-76, 1967.

[24] K. B. McGowan, M. S. Kurtis, L. M. Lottman, D. Watson, and R. L. Sah, "Biochemical quantification of DNA in human articular and septal cartilage using PicoGreen and Hoechst 33258," Osteoarthritis and Cartilage, vol. 10, no. 7, pp. 580-587, 2002.

[25] J. D. Richmon, A. Sage, V. W. Wong, A. C. Chen, R. L. Sah, and D. Watson, "Compressive biomechanical properties of human nasal septal cartilage," American Journal of Rhinology, vol. 20, no. 5, pp. 496-501, 2006.

[26] A. C. Chen, W. C. Bae, R. M. Schinagl, and R. L. Sah, "Depthand strain-dependent mechanical and electromechanical properties of full-thickness bovine articular cartilage in confined compression," Journal of Biomechanics, vol. 34, no. 1, pp. 1-12, 2001.

[27] A. K. Williamson, A. C. Chen, and R. L. Sah, "Compressive properties and function-composition relationships of developing bovine articular cartilage," Journal of Orthopaedic Research, vol. 19, no. 6, pp. 1113-1121, 2001.

[28] W. Gubisch, M. Greulich, and K. Donath, "Experimental and clinical study on the vitality of orthotopic cartilage implants," Plastic and Reconstructive Surgery, vol. 95, no. 4, pp. 663-671, 1995.

[29] B. V. Lattyak, C. S. Maas, and J. M. Sykes, "Dorsal onlay cartilage autografts: comparing resorption in a rabbit model," Archives of Facial Plastic Surgery, vol. 5, no. 3, pp. 240-243, 2003.

[30] C. Bermueller, S. Schwarz, A. F. Elsaesser et al., "Marine collagen scaffolds for nasal cartilage repair: prevention of nasal septal perforations in a new orthotopic rat model using tissue engineering techniques," Tissue Engineering A, vol. 19, no. 19-20, pp. 2201-2214, 2013.

[31] I. Fulco, S. Miot, M. D. Haug et al., "Engineered autologous cartilage tissue for nasal reconstruction after tumour resection: an observational first-in-human trial," The Lancet, 2014.

[32] U. Vetter, W. Pirsig, G. Helbing, W. Heit, and E. Heinze, "Patterns of growth in human septal cartilage: a review of new approaches," International Journal of Pediatric Otorhinolaryngology, vol. 7, no. 1, pp. 63-74, 1984.

[33] P. Adlington, A. J. Anscombe, and J. J. Phillips, "Influence of the mode of preparation on the long-term efficacy of homologous costal cartilage implants," Journal of Laryngology and Otology, vol. 106, no. 6, pp. 511-517, 1992. 


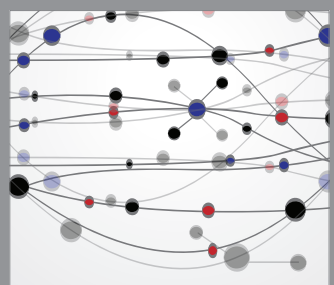

The Scientific World Journal
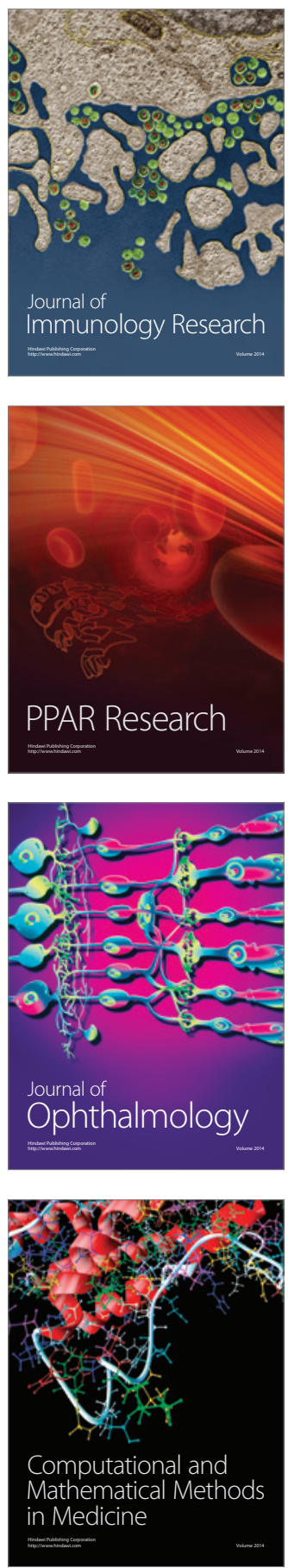

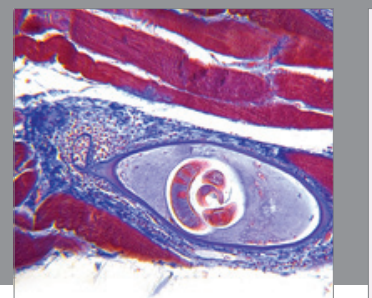

Gastroenterology

Research and Practice
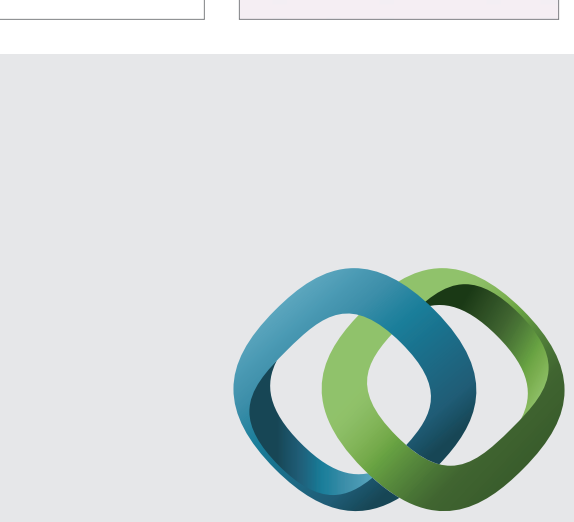

\section{Hindawi}

Submit your manuscripts at

http://www.hindawi.com
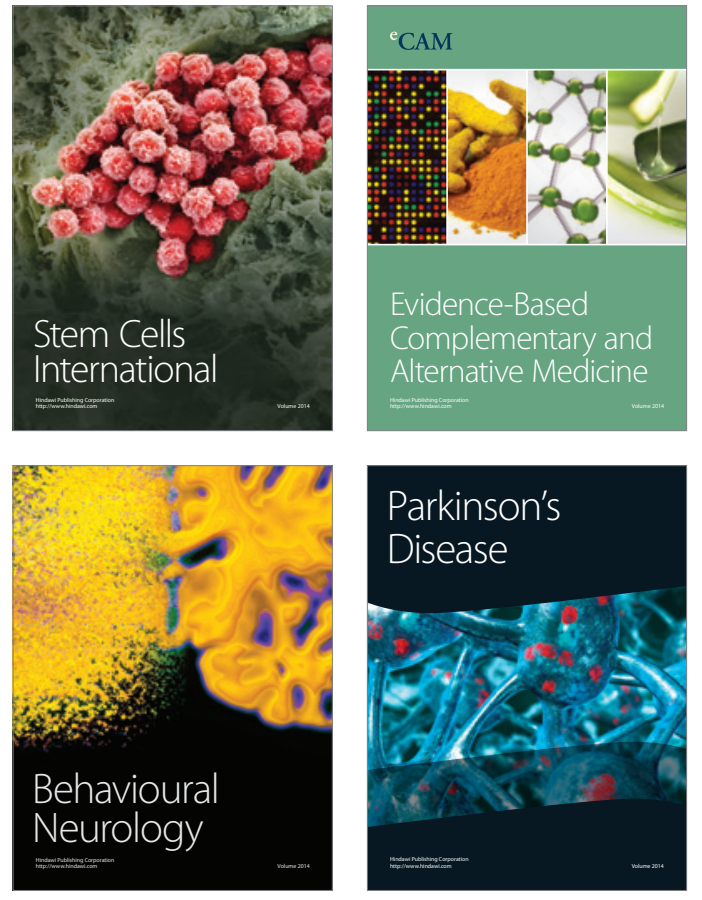
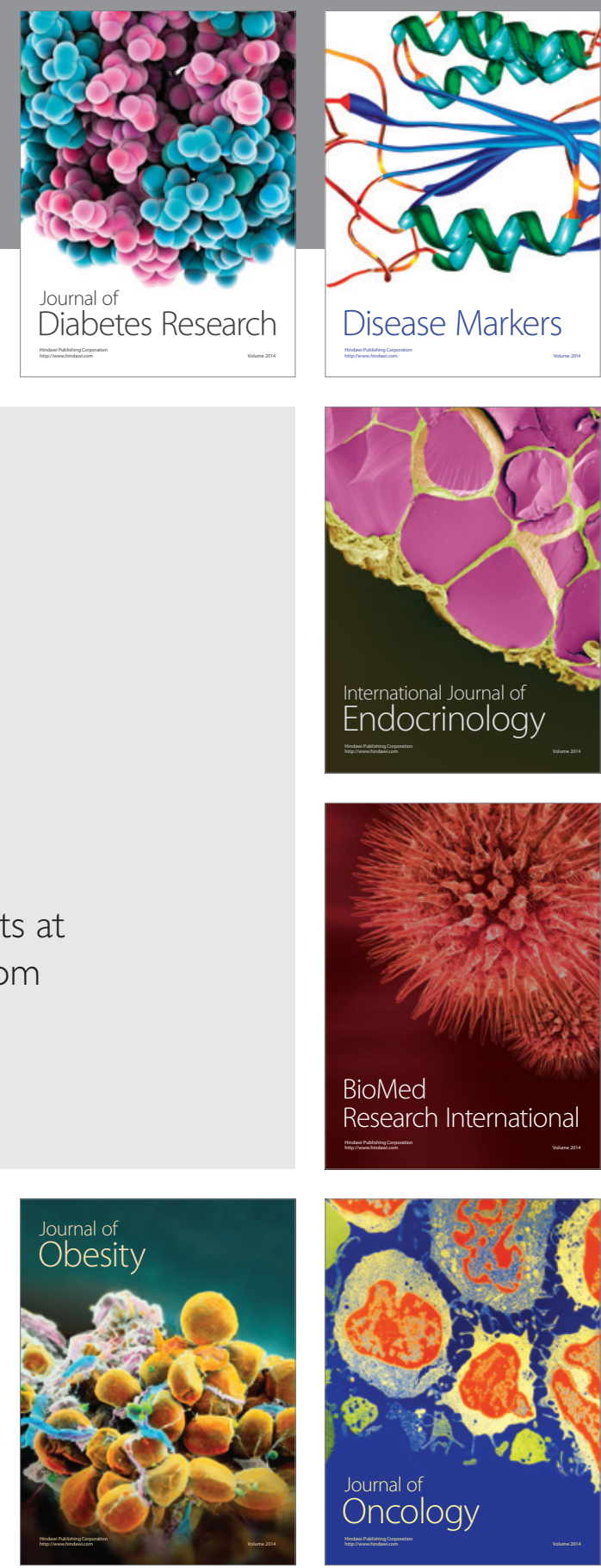

Disease Markers
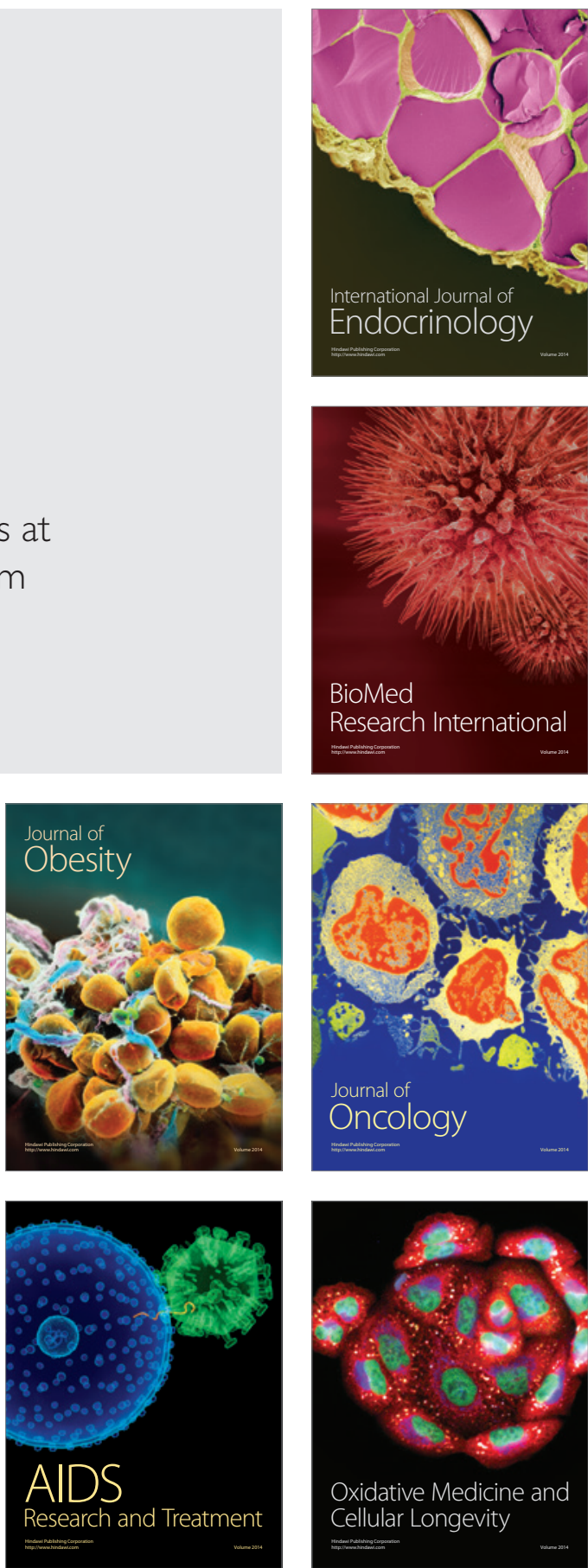